\title{
El impacto de la Covid-19 en la población mayor dependiente en España con especial referencia al sector residencial
}

\author{
The impact of COVID-19 on the elderly dependent population \\ in Spain with special reference to the residential care sector
}

Vicente Marbán Gallego (https://orcid.org/0000-0002-1825-3829) ${ }^{1}$

Julia Montserrat Codorniu (https://orcid.org/0000-0002-6798-6973) ${ }^{2}$

Gregorio Rodríguez Cabrero (https://orcid.org/0000-0002-8396-9348) ${ }^{1}$
${ }^{1}$ Departamento de Economía, Facultad de CC Económicas, Empresariales y Turismo, Universidad de Alcalá. Pza de la

Victoria 3, Alcalá de Henares. 28802

Madrid Espanha.

vicente.marban@uah.es

${ }^{2}$ Red Europea de Política

Social, Comisión Europea.

Madrid España.

\begin{abstract}
The objective of this study is to analyze the residential care crisis in Spain in the context of the COVID-19 pandemic and its impact on high mortality and abandonment of the user population. The direct, indirect and structural causes are analyzed. Specifically, precarious employment in residences over the past decade was analyzed as one of the main explanatory causes of the structural crisis of nursing homes. The theoretical focus of analysis is the comprehensive and person-centered care (CPCC) model based on the autonomy of people and the centrality of their rights. The methodology combines a quantitative analysis of employment and a qualitative analysis of documents and debates. The study concludes by proposing a comprehensive reform of long-term care that includes both a change in residential care in the form of small cohabitation units and reinforcement of care in the home and the community as a growing preference for the elderly population. An optimal combination of residential and home care is the basic proposal of this work.
\end{abstract}

Key words Nursing homes, Pandemic, Precariousness, Social and health coordination, Care reform
Resumen Este artículo tiene como objeto analizar la crisis de la atención residencial en España en el contexto de la Covid-19 y su impacto en una elevada mortalidad y el abandono de la población usuaria. Se analizan sus causas inmediatas, mediatas y estructurales. De manera específica se analiza la precariedad en el empleo en las residencias a lo largo de la pasada década como una de las principales causas explicativas de la crisis estructural de las residencias. El enfoque teórico de análisis es el modelo de atención integral y centrada en la persona (AICP) basado en la autonomía de las personas $y$ en la centralidad de sus derechos. La metodología combina el análisis cuantitativo en lo referente al empleo junto con una metodología cualitativa basada en el análisis de documentos y debates. El artículo concluye proponiendo una reforma integral de los cuidados de larga duración que incluya tanto un cambio en la atención residencial bajo la forma de pequeñas unidades de convivencia, como un reforzamiento de la atención en el domicilio y la comunidad en cuanto preferencia creciente la población mayor. La combinación óptima de la atención residencial y domiciliaria es la propuesta básica de este trabajo.

Palabras clave Residencias, Pandemia, Precariedad, Coordinación sociosanitaria, Reforma de los cuidados 


\section{Introducción}

La Covid-19 ha tenido un gran impacto en España entre marzo y junio de 2020, durante la primera oleada de la pandemia. Frente a la misma se han aplicado políticas de contención sanitaria mediante el confinamiento de la población durante tres meses, políticas económicas para hacer frente a la caída de la producción y del empleo y políticas sociales para proteger y cuidar a la población vulnerable y en situación de riesgo de exclusión social, sobre todo la población mayor, tanto la que vive en casa como en residencias.

El impacto de la Covid-19 en las personas mayores y con discapacidad que viven en residencias ha tenido una especial intensidad y dramatismo que se ha reflejado en una elevada mortalidad y un desbordamiento de la capacidad de respuesta asistencial. La tesis de este trabajo es que la causa de esto no reside sólo en la pandemia, sino que existen causas estructurales.

Este dramático impacto ha cuestionado no sólo el actual modelo de residencias para personas en situación de dependencia sino también el sistema de cuidados de larga duración implantado en España en diciembre de 2006.

En este contexto de triple crisis sanitaria, social y económica, este artículo tiene como objetivo dar respuesta a tres preguntas: cuál es la estructura sociodemográfica de las personas mayores y la correspondiente estructura de oferta de servicios para personas en situación de dependencia; en segundo lugar, si la estructura residencial en España, tenía y tiene capacidad organizativa y asistencial para hacer frente a los efectos de la Covid-19, centrando el análisis en la estructura del empleo profesional de las residencias; finalmente, qué impacto ha tenido la pandemia en el sector residencial, la razón del exceso de mortalidad, el desbordamiento de la capacidad de respuesta sociosanitaria y la crisis de gobernanza y de coordinación entre las diferentes Administraciones centrales y territoriales. Como consecuencia del análisis nos planteamos qué reformas son necesarias tanto en el sector residencial, así como en el conjunto del sistema de cuidados de larga duración (CLD), para que estos sean de calidad $\mathrm{y}$ tengan como protagonistas a las personas afectadas. Finalizamos el trabajo con una discusión de los resultados del análisis. La segunda ola de la pandemia, iniciada a finales de Agosto de 2020, ha colocado de nuevo en el punto de mira a la población que viven en residencias y la necesidad de aplicar políticas de protección sociosanitaria integrales, al mismo tiempo que se hace urgen- te la reforma integral del sistema de cuidados de larga duración en España en el contexto de la Agenda 2030.

\section{Panorámica general de la estructura de servicios para personas mayores: especial atención al sector residencial en España}

\section{Panorámica socio-demográfica de las personas mayores en España}

Según Eurostat, en 2018 las personas mayores de 65 años ya suponen en España 9,1 millones de personas $(19,4 \%$ de la población) y las proyecciones de envejecimiento para 2030 y 2050 indican que llegarán a representar el 24,1\% y 32,4\% respectivamente (UE 27: 20,3\%; 24,3\%, 29,3\%). En el caso de los mayores de 75 años representan el $9,6 \%$ y alcanzará el $11,8 \%$ en 2030 y el $18,9 \%$ en 2050 (UE27:9,7\%, 12,1\% y 17,1\%). La ratio de dependencia (15-64) se estima que pasará del $29,2 \%$ en 2018 al $37,9 \%$ en 2030 y al $59,3 \%$ en $2050(30,8 \%, 39,5 \% \text { y } 51,9 \% \text { en la UE27 })^{1}$. Según estas estimaciones, en 2050 España ocupará la cuarta posición de los países de la UE27 con mayores ratios de dependencia y de personas que superan los 65 años y los 75 años. A ello habría que añadir que España es uno de los países de la UE-27 con mayor esperanza de vida (21,6 años) y más años de vida saludable a la edad de $65(11,4$ años). La población potencialmente dependiente aumentará de 1,55 millones en 2016 a 1,99 millones en 2030 y a 3,20 millones en 2050 .

Por otra parte, hay que destacar otros condicionantes como son los cambios en la estructura de organización y provisión de los cuidados a personas mayores y personas dependientes que se ha producido en las últimas décadas. La reorganización de la oferta tradicional de cuidados debido al menor tamaño de las familias y la creciente participación de la mujer en el mercado laboral ${ }^{2}$ han potenciado la oferta de servicios formales de cuidados. Como consecuencia, se ha reducido la población cuidadora informal y se ha incrementado el volumen de horas de cuidados a las personas dependientes. El porcentaje de población mayor de 16 años que realiza tareas de cuidados de personas dependientes es del 3,4\% (1.312.400 personas; $4,2 \%$ mujeres; $2,5 \%$ varones) (EU27: 6,3\%). España es al mismo tiempo el primer país de la UE27 en lo que se refiere al porcentaje de personas que afirma dedicar más de 20 horas semanales de cuidados (53\%) (EU27: $22 \%)^{3}$, lo que supone casi 3 horas diarias de trabajo que, en general, es no remunerado. 


\section{La estructura de servicios para personas mayores}

La estructura de servicios para personas mayores y personas dependientes está integrada dentro de los servicios sociales de cada Región o Comunidad Autónoma, si bien existe un catálogo de servicios de referencia común para todo el Estado.

Los Servicios Sociales dirigidos a las personas mayores en España se pueden englobar en cuatro grandes categorías como son los servicios de atención domiciliaria, servicios de atención diurna, servicios de participación social y los servicios de atención residencial ${ }^{4}$ (Tabla 1).

Dentro de estos servicios destacan los servicios de teleasistencia y la ayuda a domicilio. La teleasistencia se ofrece a las personas mayores o a las personas con un grado moderado de dependencia que viven en casa para resolver situaciones de emergencia, inseguridad, soledad o aislamiento. Es el servicio que cuenta con un número más elevado de personas usuarias ( 942.446 personas, $10,41 \%$ de las personas mayores). La mayoría de los usuarios son mayores de 80 años (67\%). El servicio de ayuda a domicilio, que da respuesta a las necesidades básicas de la vida diaria de las personas mayores en el domicilio, atiende al 5\% de las personas de 65 y más años. El $69 \%$ de estas supera los 80 años de edad.

Los servicios de Atención Diurna incluyen sobre todo centros de día orientados a la atención psicosocial de personas mayores en situación de dependencia y suponen un servicio de respiro para los cuidadores. A 31 de diciembre de 2018, atendió al 1,1\% de las personas mayores (96.500 plazas distribuidas en 3.603 centros).

Finalmente, los servicios de cuidados residenciales, objeto de este análisis, ofrecen alojamiento

Tabla 1. Índice de cobertura por tipo de Servicios Sociales para personas mayores en España.

\begin{tabular}{lc}
\hline \multicolumn{1}{c}{ Tipo de Servicio Social } & $\begin{array}{c}\text { Índice de cobertura } \\
\text { (plazas/población } \\
\geq \mathbf{6 5} \text { años) }\end{array}$ \\
\hline Teleasistencia & $10,41 \%$ \\
Ayuda a domicilio & $4,99 \%$ \\
Centros de Día & $1,07 \%$ \\
Centros de mayores & $46,3 \%$ \\
Servicios de atención & \\
residencial & $4,32 \%$ \\
. Centros Residenciales & $4,21 \%$ \\
. Viviendas para mayores & $0,11 \%$ \\
\hline Fuente : Elaboración propia a partir de IMSERSO (2019).
\end{tabular}

y manutención a las personas mayores de manera permanente o temporal. Destacan las residencias y las viviendas para mayores. En las residencias se ofrece alojamiento y atención especializada a aquellas personas mayores que por su situación familiar, económica y social, así como por sus limitaciones de autonomía personal, no pueden ser atendidas en sus domicilios. Representan más del $97 \%$ de los servicios de cuidados residenciales. En la actualidad, España cuenta con 4,2 plazas de residencia por cada 100 personas mayores; en total, 381.158 plazas en $2018^{4}$.

\section{Los servicios de cuidados residenciales en España}

Los servicios sociales para personas mayores son competencia exclusiva de las Comunidades Autónomas cada una de las cuales tiene su propia Ley de Servicios Sociales. No existe una Ley de Servicios Sociales para todo el Estado.

Las CCAA se encargan de la acreditación, el registro y el control de calidad de todos los centros sociales de su ámbito territorial, de acuerdo con la Ley 39/2006, de 14 de diciembre, de Promoción de la Autonomía Personal y Atención a las personas en situación de dependencia (en adelante LAPAD).

Los criterios mínimos para todo el Estado en lo que respecta a la ratio de cuidadores por usuario, las cualificaciones del personal y los recursos materiales, el equipo y la documentación de los centros de atención acreditados se establece a través del Consejo Interterritorial del Sistema para la Autonomía y Atención a la Dependencia (CISAAD) en el que están representados las Administraciones central y regional. En el caso de las residencias de personas mayores dependientes se establece una ratio media de 0,41 por cada residente. Dentro de esa ratio debe haber 0,28 cuidadores, gerocultores o similares para grado III y 0,27 para grado II. Pero como analizamos en la siguiente sección el problema no son los ratios, sino el déficit en la calidad del empleo.

En la práctica, la legislación estatal se adapta a la legislación regional sobre servicios sociales. Como resultado, existe una amplia diversidad entre los reglamentos y planes de calidad. No hay un único sistema de gestión de residencias, lo que obliga a que las comparaciones entre las regiones deban ser cautelosas.

Las residencias de mayores no se consideran centros sanitarios y no están integradas en los sistemas sanitarios autonómicos. Salvo algunos centros de carácter sociosanitario, la mayoría no son centros medicalizados y acceden al sistema 
nacional de sanidad en las mismas condiciones que el conjunto de los ciudadanos.

Los servicios sociales públicos para personas mayores se prestan directamente a través de una red pública de centros regionales y municipales (representan en torno al 30\% del total de residencias en España) o bien a través de centros privados acreditados (mercantiles y sin fin de lucro) subvencionados por el sector público (suponen el $70 \%$ de las residencias). La gestión privada sin fin de lucro está siendo desplazada de manera creciente por el sector mercantil, en el que predomina el capital de fondos de riesgo.

\section{Génesis de la crisis del sector residencial}

El impacto del Covid 19 en la morbilidad y mortalidad de las personas mayores, que analizamos en la sección tercera, ha sido especialmente mortífero en espacios cerrados donde confluyen un alto grado de contacto social y personas de avanzadas edad. Los factores de la coyuntura de la pandemia no pueden soslayar la importancia de factores estructurales que caracterizan al sector residencial español. Estos factores se han manifestado en toda su dimensión y constituyen gran parte de la explicación de la crisis del sector residencial en la primavera de 2020. Entre tales factores hay que diferenciar los de tipo demográfico y asistencial, los normativos y administrativos y, finalmente, los referentes a la estructura laboral de las residencias. Este último factor es central en el análisis que aquí presentamos ya que afecta a la calidad asistencial.

-Entre los factores de tipo demográfico y sanitario hay que destacar, por una parte, el sobre envejecimiento en las residencias: la población de 80 y más años supone el $79 \%$ de toda la población que vive en residencias ${ }^{1,5}$. Este sobre envejecimiento hace especialmente vulnerable a este colectivo ante situaciones de crisis sanitaria. Por otra parte, el alto nivel de ocupación: aunque faltan de mecanismos de recopilación de datos sobre los recursos de atención residencial disponibles $^{6}$ y se desconoce con exactitud el nivel de ocupación de las residencias, se estima que en 2019 viven por término medio 322.180 personas de 65 y más años en residencias de mayores, lo que implica que un $86 \%$ del total de plazas existentes en las residencias estarían ocupadas ${ }^{5}$. Este alto nivel de ocupación se debe en cierto modo a la insuficiencia de plazas públicas y concertadas de atención residencial ${ }^{6}$. Además, existe una elevada concentración de las plazas en grandes residencias: el modelo predominante de servicios de cuida- dos residenciales en España es el de residencias de gran tamaño, con una alta concentración de las plazas en grandes residencias e insuficientes ratios de trabajadores por residente. El 50\% de las plazas se concentra en centros con más de 100 residentes y el $29 \%$ en centros entre 50 y 100 residentes. Finalmente, hay que destacar la baja sanitarización de los centros residenciales. La atención sanitaria en los centros residenciales suele prestarse derivando a los residentes a los centros de atención primaria o en los hospitales de la red del Sistema Nacional de Salud. Solo un grupo reducido de residencias de personas mayores tienen un carácter sociosanitario con una atención médica y de enfermería más amplia. Por lo general, antes de la pandemia, existía una adecuada coordinación entre las residencias y los centros de la red del Sistema Nacional de Salud. No obstante, los ajustes producidos en el sistema sanitario desde la crisis económica 2008-2015, con la consiguiente reducción de recursos disponibles en el sistema sanitario, junto a la baja eficacia del sistema de alertas tempranas y la falta de previsión de las administraciones públicas sobre el alcance de la pandemia, han acelerado la saturación de los centros hospitalarios y obligado, en algunas regiones, a recurrir al triaje médico en perjuicio de las personas de edades más avanzadas y con patologías previas.

-Entre los factores normativos y administrativos hay que destacar los siguientes. En primer lugar, el insuficiente número de inspecciones: Según el Defensor del Pueblo ${ }^{6}$, aunque las regiones están dando cada vez una mayor importancia a la inspección de centros, estas siguen siendo insuficientes dado el gran número de residencias existente y los diferentes modelos de gestión. La falta de inspecciones también ha supuesto que no se haya supervisado adecuadamente la calidad asistencial, lo que ha agravado la situación de aquellas personas mayores que presentaban carencias nutricionales cuando ha llegado la pandemia. En segundo lugar, la dispersión normativa entre las regiones sobre centros residenciales sobre los requisitos de autorización y acreditación entre las regiones, y un deficiente mecanismo de recopilación de datos, siguen dificultando el diseño de las estrategias más adecuadas para la atención residencial ${ }^{6}$.

-El tercer factor estructural explicativo de la crisis del factor residencial es el que se refiere a la estructura y calidad del empleo. En las residencias de mayores el principal factor de producción son los empleados que atienden a las personas, especialmente, el personal de atención directa (cui- 
dadores, enfermería, de animación, entre otros). La calidad de la atención a las personas usuarias dependerá, en gran parte, de las condiciones de trabajo de su personal.

Durante la crisis sanitaria de la Covid-19 se han evidenciado las dificultades de atención en las residencias por falta de suficientes cuidadores y de profesionales cualificados, como en el caso de enfermería, pero también se ha hecho evidente la precariedad de las condiciones laborales de dicho personal. Aquí hacemos referencia a dos aspectos fundamentales: los tipos de contratos laborales y las retribuciones salariales. Estos son resultado de un trabajo de investigación sobre el empleo en los establecimientos residenciales para mayores y con discapacidad física ${ }^{7}$.

En el sector de los servicios residenciales para mayores, el elevado número de contratos de duración determinada (temporales), con pocos días o pocas horas de trabajo, junto con los contratos indefinidos a tiempo parcial, muestra la incorreción en utilizar el indicador "número total de empleados" para describir el empleo de un centro, ya que no es indicativo de la fuerza de trabajo realmente utilizada. Por ello, se ha recurrido al concepto de empleo efectivo, el cual mide el personal equivalente a tiempo completo de acuerdo con las horas anuales establecidas en el convenio colectivo. Para ello se tiene en cuenta, tanto el número de días con contrato, como el número de horas contratadas de cada uno de los trabajadores. Aproximadamente, el número total de empleados al año se reduce en casi la mitad (42\%) al transformarse en empleo efectivo. Así, en 2018, el número de empleados es de 153.625 mientras que el "empleo efectivo" es de 89.248.

Una de las principales características laborales es la elevada temporalidad del empleo y su baja calidad. En 2018, más del 25\% (26.8\%) del empleo efectivo anual es realizado por personal con contratos temporales, lo cual demuestra que hay una parte importante del trabajo ordinario que es realizado por trabajadores que no están en la estructura fija de la empresa.

El 95\% de los contratos de duración determinada se agrupan en tres modalidades de contrato ("obra o servicio", "interinidad" y "eventual”) permitiendo que un mismo trabajador pueda alargar los días trabajados al año enlazando contratos de diferentes modalidades o varias veces en el mismo tipo de contrato siempre que no supere la limitación impuesta por las normas. Los contratos temporales de este grupo tienen la peculiaridad de que, además de ser de duración determinada no tienen derecho al plus de antigüedad, ni disfrutan de cualquiera de las otras ventajas de los contratos indefinidos como, por ejemplo, la formación o la participación en la organización de tareas, entre otras.

En el conjunto de las tres modalidades especificadas de contrato temporal la media de días con contrato al año es de casi cuatro meses $(3,71)$ y, la media del número de contratos al año por trabajador es de 2,38. Ello refuerza la idea de que los contratos temporales no se utilizan solo para cubrir bajas temporales del personal plantilla o reforzar momentos puntuales de sobrecarga de trabajo, sino que parecen estar destinados a complementar el trabajo del personal de plantilla.

Casi el 90\% del empleo efectivo es realizado por mujeres. Ello muestra que los trabajos de cuidados están altamente feminizados. Este es un factor común en los diferentes tipos de organizaciones proveedoras de servicios residenciales para mayores, con independencia de que sean organizaciones lucrativas (sociedades de capital), organizaciones no lucrativas (asociaciones, fundaciones) $\mathrm{u}$ organizaciones públicas (corporaciones locales $\mathrm{u}$ otros organismos públicos), aunque las instituciones religiosas y las corporaciones locales son las que encabezan el ranking.

El segundo factor que muestra la baja calidad del empleo en los centros residenciales de mayores es la gran concentración de trabajadores en las categorías profesionales bajas según la clasificación de la Seguridad Social. Estas categorías son indicativas de las características del puesto que ocupan y de la retribución salarial que les corresponde más que de la cualificación profesional de los trabajadores.

Los resultados muestran que, en promedio, el $80 \%$ de los empleos están encuadrados en las categorías profesionales más bajas -auxiliares administrativos, oficiales, subalternos y peones $\mathrm{y}$ asimilados. Ello da idea del nivel de titulaciones exigidas al personal para trabajar en dichos centros y de la escasa proporción de trabajadores con niveles profesionales, reconocidos, de grado medio o superior. El resto de los trabajadores se distribuyen del siguiente modo: un 13\% en el grupo de categoría media -titulados de grado medio y cargos de responsabilidad media- y un 7\% en el grupo de categoría alta -titulados superiores, directores y altos cargos.

El 80\% de los empleados en los establecimientos residenciales para personas mayores perciben, como media, un salario bruto mensual de 1.184 euros, el cual, se estima, se reduce a un salario neto mensual inferior a 1.000 euros una vez que se han realizado las retenciones del impuesto sobre la 
renta de las personas y de la cotización de la Seguridad Social a cargo del trabajador. Este importe está en el límite del salario mínimo interprofesional. Sin embargo, cabe concluir que, aunque los salarios están dentro de los márgenes que fija la Ley, no se corresponden con el esfuerzo y dedicación que deben realizar estos trabajadores para atender a unos usuarios muy vulnerables.

La Tabla 2 muestra los resultados de los tres grandes grupos según la categoría profesional. Los salarios de los trabajadores incluidos en la "categoría alta" probablemente no se recogen "correctamente" ya que están limitados por el "tope máximo" de cotización a la Seguridad Social.

La brecha de género de los empleados encuadrados en la categoría baja es muy pequeña $(1,5 \%)$. Se intuye que, cuando los salarios son bajos, no hay margen para disminuirlos, mientras que en las categorías media y alta la brecha de género aumenta alcanzando cifras bastantes similares en estos dos grupos, $7,5 \%$ y $7,1 \%$, respectivamente. Por otra parte, se percibe que hay diferencias salariales entre los diferentes tipos de contratos; así, los asalariados con contrato de duración determinada perciben un 4,2\% menos que los que tienen un contrato indefinido. La diferencia es más pronunciada en los hombres que en las mujeres ( $13,3 \%$ en hombres y $2,7 \%$ en mujeres).

En resumen, la calidad de la atención a las personas mayores depende en buena parte de la calidad de las condiciones laborales de los trabajadores. En la actualidad estas se caracterizan por un elevado grado de precarización laboral.

\section{El impacto de la crisis del COVID19 en el sector residencial para personas mayores}

El impacto diferencial de la pandemia y de la mortalidad sobrevenida en las residencias de personas mayores y con discapacidad en España estriba tanto en causas inmediatas (bloqueo de acceso al sistema hospitalario y selección adversa de personas mayores y desbordamiento de la capacidad de respuesta asistencial de este servicio), como mediatas (crisis del modelo de gobernanza) y estructurales (analizadas estas en la sección anterior).

La literatura reciente ha analizado las diferentes dimensiones de la crisis de las residencias diferenciando en general los tres tipos de causas ${ }^{8-11}$.

La Covid-19 ha tenido un impacto sanitario diferencial en las personas mayores con multipatologías, tanto las que viven en residencias como en sus domicilios. Siendo esto cierto se ha producido, aunque no de manera generalizada, un triaje o selección adversa de las personas mayores que, hasta la llegada abrupta de la pandemia, accedían normalmente al sistema hospitalario. El bloqueo de facto en el acceso al sistema hospitalario, unido a la limitada capacidad de atención sanitaria en las residencias, ha supuesto una sobremortalidad del colectivo que vive en este tipo de servicios, con cierta excepción en el caso de las residencias sociosanitarias o especializadas. No se trata tanto de un bajo nivel de medicalización de las residencias sino de una insuficiente capacidad de atención sanitaria para hacer frente a emergencias como la Covid-19. A esto hay que añadir el desbordamiento de la capacidad general de respuesta de las residencias, basada fundamentalmente en los cuidados por parte de un personal profesional precarizado, tal como hemos visto en la sección anterior, infradotados de equipos de protección individual (EPIs), de medios y técnicas y sin reemplazo en caso de contagio.

Otra causa a tener en cuenta es la crisis de la gobernanza del sistema de servicios sociales. Las residencias son competencia de cada Comunidad Autónoma o región y en este sentido la respuesta ha variado en función de las políticas y arreglos

Tabla 2. Estimación del salario mensual bruto y neto por categorías profesionales (2018).

\begin{tabular}{lcccccc}
\hline & \multicolumn{4}{c}{ Salario bruto mensual } & \multicolumn{2}{c}{ Salario neto mensual } \\
\hline & $\begin{array}{c}\text { Total } \\
\text { individuos (\%) }\end{array}$ & $\begin{array}{c}\text { Hombre } \\
\text { (euros al mes) }\end{array}$ & $\begin{array}{c}\text { Mujeres } \\
\text { (euros al mes) }\end{array}$ & $\begin{array}{c}\text { Hombre } \\
\text { (euros al mes) }\end{array}$ & $\begin{array}{c}\text { Mujeres } \\
\text { (euros al mes) }\end{array}$ & $\begin{array}{c}\text { Brecha de } \\
\text { género }\end{array}$ \\
\hline Categoría alta & $13,7 \%$ & 1.828 & 1.699 & 1.462 & 1.359 & $-7,10 \%$ \\
Categoría media & $6,7 \%$ & 1.484 & 1.373 & 1.187 & 1.098 & $-7,50 \%$ \\
Categoría baja & $79,6 \%$ & 1.201 & 1.182 & 961 & 946 & $-1,50 \%$ \\
Total & & 1.350 & 1.260 & 1.080 & 1.008 & $-6,70 \%$ \\
\hline
\end{tabular}

Nota: Salario mensual por 14 pagas.

Fuente: Montserrat ${ }^{7}$ a partir de la Muestra Continua de Vidas Laborales (MCVL) 2018. 
institucionales de cada territorio. El "Estado de Alarma" aplicado en España entre el 14 de Marzo y el 21 de Junio de 2020 no ha supuesto la aplicación de una política común como respuesta ante la crisis de las residencias. Por el contrario, ha tenido lugar una dispersión o reacción desigual en función de los modelos de atención residencial y del tipo de relación entre el sector público, minoritario, y el sector privado, mayoritario. Por otra parte, y una vez más, la crisis ha evidenciado el déficit de la coordinación entre los servicios sociales y de sanidad.

Como consecuencia de las dos causas mencionadas se ha producido una sobremortalidad de la población mayor, sobre todo la que vive en las residencias. Como se observa en la Tabla 3, el exceso de mortalidad en la población mayor de 65 años ha sido espectacular comparado con la población menor de 65 años. No todo el exceso de la mortalidad se explica por la Covid-19, pero es seguramente la causa más importante. Hasta el inicio de 2021 no se sabrá con precisión la población fallecida y las causas de defunción. En el período crítico de la pandemia, de marzo a mayo de 2020, de todo el exceso de mortalidad el 94,7\% corresponde a la población mayor de 65 años o, de otro modo, el 74,2\% del exceso de mortalidad correspondió a la población mayor de 74 años.

En cuanto a la población mayor de 65 años que ha "solicitado la prestación social" del sistema nacional de autonomía y dependencia (SAAD) la mortalidad observada ha sido de 81.232 personas frente a la mortalidad esperada de 51.369 personas entre los meses de marzo y mayo de 2020, lo que resulta en un exceso de mortalidad de 31.263 personas, es decir, un $60,9 \%$ de exceso. De este exceso de mortalidad el $83,1 \%$ tiene 80 y más años, particularmente superior en las mujeres. De la "población perceptora de la prestación social “ durante esos tres meses fallecieron 57.469 personas, 25.819 más de lo esperado, es decir, un exceso de mortalidad del $80,1 \%$; de dicho exceso de mortalidad el $84,2 \%$ tienen 80 y más años ${ }^{12}$.

La cuantificación de la mortalidad de la población que vive en residencias es objeto aún de debate al no estar debidamente centralizada la información y no disponer de indicadores rigurosos tal como señala el grupo de investigación GTM $^{9}$. Hay estimaciones a partir de la información de las Comunidades Autónomas y del Ministerio de Sanidad ${ }^{13}$. No toda mortalidad en residencias puede ser atribuida a la Covid-19, aunque si "asociada” a la misma en un gran número de casos. Así, a fecha de 28 de mayo, según la International el Long Term Care Policy Network de la LSE, la población infectada por Covid-19 era de 237.906 personas de los cuales habían fallecido 27.119. De este colectivo 19.194 personas que vivían en residencias habían fallecido por la Covid-19 o síntomas asociados, es decir, el 70,8 $\%$. La vulnerabilidad de los residentes, personas muy mayores, la precariedad en el empleo asistencial, la orientación mercantil de la gran mayoría de la actividad residencial, la crisis de la coor-

Tabla 3. Exceso de Mortalidad sobrevenida debido a la Covid-19 en dos períodos de impacto, agudo y de bajo impacto.

\begin{tabular}{lcccc}
\hline \multicolumn{5}{c}{ Período 10.3.2020 a 9.5.2020 } \\
\hline \multicolumn{1}{c}{ Población } & Observada & Estimada & Exceso Mortalidad & Exceso Mortalidad \% \\
\hline Todos & 111.253 & 67.697 & 43.556 & 64,3 \\
Hombres & 55.815 & 34.180 & 21.636 & 63,3 \\
Mujeres & 54.377 & 32.833 & 21.544 & 65.6 \\
Edad $<$ 65 & 11.773 & 9.521 & 2.252 & 23,7 \\
Edad 65-74 & 14.438 & 9.403 & 5.035 & 53,5 \\
Edad $>$ 74 & 85.042 & 48.819 & 36.223 & 74,2 \\
\hline \multicolumn{1}{c}{ Población } & Período & $\mathbf{2 7 . 7 . 2 0 2 0}$ a $\mathbf{1 5 . 8 . 2 0 2 0}$ & \\
\hline Todos & 22.763 & Estimada & Exceso Mortalidad & Exceso Mortalidad \% \\
Hombres & 11.146 & 10.176 & 2.540 & 12,6 \\
Mujeres & 11.598 & 9.851 & 970 & 17,7 \\
Edad $<$ 65 & 3.205 & 3.076 & 1.747 & 4,2 \\
Edad 65-74 & 3.016 & 2.857 & 130 & 5,6 \\
Edad $>$ 74 & 16.545 & 14.384 & 159 & 15,0 \\
\hline
\end{tabular}

Fuente: Vigilancia de la Mortalidad Diaria. Centro Nacional de Epidemiología (ISCIII). www.isciii.es. 
dinación entre servicios sociales y sanitarios, la falta de protección adecuada y de preparación de los propios profesionales, así como la ausencia de planes de contingencia internos y externos, son factores a destacar en la explicación del exceso de mortalidad y el aislamiento que han sufrido los residentes a modo de confinamiento total. Médicos sin Fronteras ${ }^{14}$ estima, a fecha de 20 de junio, que la población fallecida en residencias es de 27.354 personas, el $69 \%$ de las personas fallecidas por la Covid-19, porcentaje muy similar al antes mencionado. Las residencias han sido una trampa mortal para la población más vulnerable, en muchos casos abandonados a su suerte.

En cuanto a la crisis estructural del modelo residencial, las residencias tuvieron que asumir de facto una responsabilidad para la que no estaban preparadas, dotadas ni protegidas, con consecuencias desastrosas para residentes, personal, equipos de gestión y familiares ${ }^{14}$. Las residencias son necesarias, lo que es evidente para expertos en atención residencial. Pero la Covid-19 ha puesto de manifiesto los déficits y límites del actual modelo de cuidados en residencias. Se ha actuado tarde, poco y mal ${ }^{14}$ por parte de las autoridades responsables a pesar de cierto esfuerzo de coordinación por parte del gobierno central ${ }^{15}$.

Existe un amplio consenso entre los actores públicos y corporativos, así como del Tercer Sector, sobre los límites del actual modelo residencial. Del reciente debate cabe destacar las líneas de reforma y mejora en defensa de la salud y autonomía de las personas que viven en residencias, así como una apelación a un modelo de atención residencial integral, centrado en las personas y basado en sus derechos individuales, vivan en residencias o vivan en su domicilio.

En este sentido se han posicionado el Defensor del Pueblo, con recomendaciones muy precisas $^{16}$ a partir de las quejas de familiares de usuarios de residencias, la Sociedad Española de Geriatría y Gerontología $a^{17}$ y, en general, las organizaciones del Tercer Sector y con discapacidad y centros de estudios ${ }^{18}$. Entre las líneas de reforma se destacan las siguientes: mejora efectiva de la coordinación entre el sector de servicios sociales y el sanitario; suplir con eficacia las bajas laborales de los trabajadores de las residencias de mayores para poder garantizar el cuidado adecuado; garantizar la información continua, y al menos diaria, a la persona designada por la familia del residente sobre su situación; permitir que los mayores no contagiados que viven en una residencia puedan volver con sus familias de forma voluntaria y temporal y sin pérdida de la plaza durante esta crisis del coronavirus; adoptar protocolos, en los casos de estado clínico terminal, que permitan facilitar la despedida al menos a un miembro de la familia, con el fin de tener un proceso de muerte lo más humanizado y digno que sea posible, cumpliendo los requisitos de salud pública necesarios para garantizar la seguridad del resto de usuarios, trabajadores y del propio familiar. Medidas todas ellas dirigidas a evitar una situación en la que, según la SEGG ${ }^{17}$, "las personas mayores han sido discriminadas en su capacidad de acceso real a servicios sanitarios especializados y las residencias han sido estigmatizadas".

En conclusión, la crisis de la residencias ha puesto de manifiesto la necesidad de armonizar los protocolos de acreditación y control de calidad en todo el Estado; la mejora de los ratios de profesionales y de su cualificación y estabilidad laboral; el respeto por la autonomía y derechos de las personas residentes a ser informadas y participar en las decisiones que les afectan; una atención médica y de enfermería más amplia y extensa, sin menoscabo de la específica atención en las residencias sociosanitarias; el desarrollo y difusión de un sistema conjunto de buenas prácticas de atención residencial.

\section{Discusión: la necesaria reforma del sector residencial y el desarrollo de modos de vida en el entorno domiciliario y comunitario}

Las residencias para personas en situación de dependencia, sobre todo mayores de 65 años, constituyen un servicio consolidado en España, pero caracterizado por su elevada precariedad laboral, tamaños excesivos y desiguales resultados de calidad asistencial. Como resultado, constatamos un modelo general de "aparcamiento" de personas mayores y no un recurso de calidad, con excepciones destacadas.

Del análisis realizado se deducen dos tipos de debate. El primero se refiere a la mejora del sistema actual de residencias. Siendo necesarias, requieren una reforma profunda en la que el sector público debe tener un claro protagonismo en la acreditación de los centros, control de la calidad y defensa de los derechos de las personas residentes. Concretamente, es necesario mejorar aspectos estructurales del actual sistema residencial con adecuados ratios de trabajadores por residente y empleos de calidad, mayor financiación de los servicios sociales para personas mayores, más inspecciones de los centros residenciales y mejora de la coordinación sociosanitaria. Todo ello como requisitos instrumentales al servicio 
de un cambio de modelo integral que tendrá que poner mayor énfasis en la atención centrada en la persona, basada en la organización de los centros en pequeñas unidades de convivencia, con estructura y dimensión de hogar, en la que conviva un número reducido de personas mayores a las que se les proporcionan apoyos personalizados de acuerdo a sus necesidades y deseos ${ }^{6,19}$. También será necesario potenciar las viviendas adaptadas que solo representan en torno el 3\% del total de los servicios de cuidados residenciales.

Pero el debate tiene una dimensión más general: cómo deben vivir las personas mayores, sobre todo las que tienen algún tipo de dependencia. Este es el debate central: qué modelo de cuidados adoptar que garantice la preferencia de las personas, su diversidad, autonomía y derechos fundamentales. La residencia debe ser un recurso para personas con elevada dependencia y exigencia de atención sociosanitaria. Por el contrario, para personas mayores con dependencia moderada el recurso a proveer son servicios comunitarios y domiciliarios tanto instrumentales como asistenciales y emocionales. El desarrollo de este recurso en España ha sido intenso en los últimos dos decenios, pero no es suficiente para hacer frente a la demanda ni es un aún un servicio integral y centrado en la autonomía y centralidad de las personas $^{10,20,21}$.

La protección de las personas mayores dependientes en España afronta un horizonte de incertidumbre dada la dinámica del envejecimiento de la población y de los cambios en la estructura social de los cuidados. De ahí que reforzar el Estado de Bienestar ${ }^{22}$ y construir una sociedad de cuidados forman parte de la misma ecuación social.

\section{Colaboradores}

VM Gallego: trabajó en la elaboración y en la redacción final, sobre todo de las secciones 1, 2 y 4 . También participó en el diseño de la estructura y metodología del artículo, en la revisión y edición final y en la concepción y el análisis e interpretación de los datos. JM Codorniu: trabajó en la elaboración y en la redacción final de la secciones 2 y 4, en la revisión final y en la concepción y análisis e interpretación de los datos. GR Cabrero trabajó en la elaboración y en la redacción final, sobre todo en la introducción y las secciones 3 y 4. También participó en el diseño de la estructura y metodología del artículo, en la revisión de la versión enviada y en la concepción y análisis e interpretación de los datos

\section{Agradecimientos}

Este texto se llevó a cabo con el apoyo del Proyecto PECOTSAS del Plan Estatal de Investigación e Innovación Científica y Técnica 2013-2016 del Ministerio de Economía, Industria y Competitividad. También se ha beneficiado de documentos y declaraciones sobre la Covid-19 de la Fundación Pilares para la Autonomía Personal. 


\section{Referencias}

1. Pérez Díaz J, Abellán García A, Aceituno Nieto P, Ramiro Fariñas D. Un perfil de las personas mayores en España, 2020. Indicadores estadísticos básicos. Informes Envejecimiento en red no 25. Madrid: CSIC; 2020. [citado 2020 Jun 23]. Disponible en: https://bit. ly/3iCipzT

2. IMSERSO. Atención a las personas en situación de dependencia en España. Libro Blanco. Madrid: IMSERSO; 2005.

3. European Commission (EU). Ageing report 2018. Economic and budgetary projections for the $28 \mathrm{EU}$ Member States (2016-2070). Brussels: EU; 2018.

4. IMSERSO. Servicios Sociales dirigidos a las personas mayores en España. Madrid: IMSERSO; 2019. [citado 2020 Jun 23]. Disponible en: https://bit.ly/2FMOC99

5. Abellán A, Aceituno P, Fernández I, Ramiro D. Una estimación de la población que vive en residencias de mayores [Internet]. CSIC. Envejecimiento en Red. Departamento de Población. Madrid: CSIC; 2020. [citado 2020 Jun 23]. Disponible en: https://bit.ly/ 3 moRKZQ

6. Defensor del pueblo. Atención a personas mayores. Centros residenciales. Separata del Informe anual 2019. Madrid: Defensor del Pueblo; 2020a.

7. Montserrat Codorniu J. La calidad del empleo en las residencias para personas mayores. Incidencia en la gestión de la COVID-19. Zerbitzuan 2020; 73.

8. Manzano MA. Report: COVID-19 and long-term care in Spain: impact, underlying problems and initial measures. London: LTCcovid.org; 2020 [citado 2020 Sep 7]. Disponible en: https://bit.ly/3kALIDV

9. España. Ministerio de Ciencia e Innovación. Informe del GTM sobre el impacto de la COVID-19 en las personas mayores, con especial énfasis en las que viven en residencias. Madrid: Grupo de Trabajo Multidisciplinar; 2020.

10. Rodríguez Rodríguez P. ¿Qué fue de los avances en atención integral/integrada y centrada en la persona (AICP) en residencias durante la pandemia COVID19? Madrid: Dependencia.Info; 2020. [citado 2020 Sep 7]. Disponible en: https://bit.ly/3hBebaw

11. Zalakain J, Davey V, Suárez-González A. The COVID-19 on users of Long-Term Care services in Spain. London: LTCcovid, International Long-Term Care Policy Network, CPEC-LSE; 2020. [citado 2020 Sep 10]. Disponible en: https://bit.ly/2FrQogs

12. IMSERSO. MoMo en el sistema para la autonomía y atención a la dependencia (SAAD) Nota ejecutiva. Datos a 30 de junio de 2020. Madrid: IMSERSO; 2020. [citado 2020 Sep 7]. Disponible en: https://bit.ly/2FCmFAZ .

13. Pino E, Moreno J, coordinadores. Gestión Institucional y Organizativa de las Residencias de Personas Mayoresy COVID-19: dificultades y aprendizajes. Madrid: CSIC; 2020.
14. Médicos sin Fronteras (MSF). Poco, tarde y mal. El inaceptable desamparo de los mayores en las residencias durante la Covid-19 en España. Madrid: Médicos sin Fronteras; 2020.

15. España. Ministerio de Derechos Sociales y Agenda 2030. Documento técnico de recomendaciones de actuación desde los servicios sociales de atención domiciliaria ante la crisis por COVID-19. Madrid: Ministerio de Derechos Sociales y Agenda 2030; 2020. [citado 2020 Sep 7]. Disponible en: https://bit.ly/3ixifKe.

16. Defensor del Pueblo. Recomendaciones sobre residencias de mayores, atención sanitaria e información emergencia Covid-19. Madrid: Defensor del Pueblo; 2020. [citado 2020 Jun 23]. Disponible en: https://bit. ly/3c5gnpD .

17. Sociedad Española de Geriatría y Gerontología (SEGG). Propuestas covid-19 de la geriatría y gerontología. Madrid: SEGG; 2020. [citado 2020 Sep 7]. Disponible en: https://bit.ly/32AmIWS

18. Envejecimiento en Red. Ante la crisis de la Covid-19: una oportunidad para un mundo mejor. Declaración en favor de un necesario cambio en el modelo de cuidados de larga duración de nuestro país. Madrid: CSIC; 2020. [citado 2020 Sep 7]. Disponible en: http://www.acpgerontologia.com .

19. Fundación Pilares. La atención centrada en la persona en los servicios gerontológicos. Modelos de atención y evaluación. Madrid: Fundación Pilares. Colección Estudios de la Fundación 3; 2015. [citado 2020 Sep 7]. Disponible en: https://bit.ly/2ZHUNTf

20. Rodríguez Rodríguez P. Fundación Pilares, centrados en las necesidades de los mayores dependientes. Entrevista a Pilar Rodríguez. Revista 65 y más 2020; 3. [citado 2020 Sep 7] Disponible en: https://bit.ly/3iFAqgT

21. Ribera Casado JM. Covid-19 y residencias de ancianos: algunas reflexiones. Revista de la Academia $\mathrm{Na}$ cional de Medicina de España 2020; 137:107-111.

22. Greve B. Preparing welfare states in the age of COVID-19. En: Pamphlet. 12 perspectives on the pandemic. Berlín: De Gruyter Social Sciences; 2020. p. 25-30.

Artículo presentado en 22/09/2020

Aprobado en 22/09/2020

Versión final presentada en 24/09/2020

Editores chefes: Maria Cecília de Souza Minayo, Romeu Gomes, Antônio Augusto Moura da Silva. Editora Associada da área de Saúde do Idoso: Joselia Oliveira Araújo Firmo 